\title{
RECUPERAR UNA VISIÓN UTÓPICA (ENTREVISTA COM O PROF. DR. HUGO ZEMELMAN)*
}

\author{
Célia FrazÃo Linhares** \\ M aria Ciavatta Fran Co***
}

\begin{abstract}
RESU M 0 : Esta entrevista foi concedida pelo Prof. H ugo Zemelman, em 23 de setembro de 1993, quando, na qualidade de Professor Visitante, eleministrou a disciplina "O shorizontes da razão ea produção do conhe cimento" no Curso de M estrado em Educação da U niversidade Federal Fluminense. A preocupação das entrevistadoras foi conhecer seu pensamento diante dos problemas postos pelo presente e pelo futuro de um mundo altamente tecnologizado e socialmente em deses-truturação.
\end{abstract}

Palavras-chave: Razão; Visão U tópica; Liberdade; Latino-americano; Futuro.

(E) Aquí en Brasil estamos extremamente preocupados con esa desigualdad, con esa onda de exclusión que viene tornándose cada vez más intensa. Parece que los límites del sueño de libertad se escapan de nuestras mentes. Este fin de siglo que nos prometió tanto en términos de razón, de ciencia, de escuela, nos aproximamos del próximo siglo amenazados por la desesperanza, por la apatía. En este cuadro general, ¿C uál es tu posición en torno de estas cuestiones fudamentales para la humanidad?, ¿En qué

* Hugo Zemelman é sociólogo, professor pesquisador de tempo integral do Centro de Estudos Sociológicos de El Colegio de M éxico; conferencista e professor visitante em várias universidades latino-americanas, autor de artigos e livros tais como U so crítico de la teoria (El Colegio de M éxico, 1987), De la história a la Política - La experiencia de América Latina (Siglo Veintuno, 1989); Los horizontes de la razón (Anthropos, 1992); D eterminismos y alternativas en las ciencias sociales de América Latina (coord.) (N ueva Sociedad, 1995). A entrevista será apresentada com as inicias (E) para as entrevistadoras e $(\mathrm{HZ})$ para as respostas do entrevistado.

* Doutora em Filosofia, professora titular de Políticas Públicas e docente do Programa de PósGraduação em Educação (M estrado e D outorado), da U niversidade Federal Fluminense (N iterói, RJ). E-mail: clinhar@pontocom.com.br

* Doutora em Ciências H umanas (Educação), professora titular de Trabal ho e Educação e docente do Programa de Pós-G raduação em Educação (M estrado e D outorado) da U niversidade Federal Fluminense. E-mail: ciavatta@alternex.com.br 
espacio se puede soñar el sueño de la libertad, de la solidaridad y de la organización de un nuevo mundo sin exclusión?, ¿Cómo soñar ese sueño?, ¿Es posible?

(H Z) Creo que lo importante de reconocer en este momento es precisamente que se ha perdido la capacidad de sonãr. C reo que recuperarla es una tarea ineludible, fundamental en el presente. En el ámbito estricto de quienes están preocupados en construir un conocimiento que tenga un sentido de recuperación de la dignidad humana no puede ser ajeno en ese esfuerzo la recuperación de un sentido fundamental del conocimiento, como es el de vislumbrar un futuro posible. Pero para lograr esto se requiere recuperar lo que se ha perdido junto con el sueño, se ha perdido la capacidad de pensar utópicamente. Recuperar la utopía en este momento creo que es consustancial a la construcción de conocimiento y al pensar mismo, porque es la forma que el hombre tiene de ver su propia circunstancia distanciándose de ella, y al distanciarse no quedarse atrapado como en una cárcel. $\mathrm{H}$ ay mucho discurso que expressa este sentimiento de prisión. Un discurso que continúe atado a ciertas lógicas de pensar muy deterministas y muy mecánicas no ayuda, por mucho que profundice en el conocimiento de la realidad, a recuperar un sentido emancipatorio porque carece de utopía. Y si esta utopía en definitiva no está ausente, en todo caso es una utopía que no es diferente a aquella que se pretende imponer.

Yo creo que el problema que tu disenãs supone en el ser humano, como lo han señalado muchas personas, colocar en la discusión problemas dolorosos, pero fundamentales. El primero de los cuales es tomar conciencia de que por diversas razones el hombre ha alcanzado hoy en día, en su máximo desarrollo civilizatorio, una debilidad intrínseca hasta el punto de que puede colocarse en el límite de su propia muerte. Creo que temas como el de la pérdida del instinto de conservación, son temas que se han discutido poco y que de alguna manera creo que habría que colocar en el plano de la discusión, sobre todo si queremos recuperar una esperanza de futuro. Y curiosamente, la pérdida de ese instinto de conservación por parte del hombre está muy fuertemente asociada a su propio progreso técnico e intelectual. Ahí hay como una contradicción autodestructiva: mientras más capacidad tiene el hombre de enfrentar su realidad, menos instinto de preservarse.

Las lógicas económicas que vemos en este momento dominando el escanario de los países latinoamericanos, creando esas desigualdades y esas marginalidades crecientes son una demostración de lo que les digo. Evidentemente eso define desafíos importantes. El desafío está en volver 
a repensar viejas ilusiones como es la de hombre constructor de su destino, la del hombre constructor de vida, el hombre que controla la adversidad. Creo que eso es algo que en este momento está en cuestionamiento, creo que la mayor capacidad del hombre, en lugar de colocarlo frente al mundo y frente a la naturaleza, en condiciones de poder dignificarse, reconoce mucho más su capacidad, no digo sólo destructiva de lo que lo rodea, como la naturaleza, sino también autodestructiva.

En ese contexto yo ubicaría algunos temas más puntuales de tu pregunta. Uno de ellos sería el tema de la libertad, pero la libertad no en un sentido metafísico, sino la libertad como la posibilidad de realización de la persona. Cuando constatamos que la sociedad moderna, burocrática y compleja, sometida a procesos que se han sostenido, que son espectaculares, reduce el espacio del ser humano cada vez más, donde el individuo es cada vez menos importante, donde incluso el concepto de persona pierde relevancia, cuando la persona humana, el ser humano, el individuo queda cada vez más sometido a una lógica inexorable que lo controla, que deviene de los avances tecnológicos. Estamos hablando de un individuo que está encontrando en algunos espacios mínimos la salvación mínima de su condición de ser pensante. Porque el resto de los espacios de la sociedad están cada vez más controlados por una máquina infernal que tiene una lógica que lo aplasta. Cuando hablamos de libertad, estoy tratando de vincular el concepto de libertad a un sentido último que tiene la construcción del conocimiento. $Y$ ese sentido último, en mi opinión, está en la posibilidad de leer, de interpretar, y por lo tanto de poder relacionarse con la realidad que nos condiciona desde una exigencia de futuro.

Esa exigencia de futuro es una exigencia valórica, axiológica, supone una visión de futuro, pero hemos perdido la visión de futuro. No se trata tampoco de una escatología, se trata más bien de colocarse en la postura desde una visión de futuro que nos permita leer la realidad que nos está en este momento condicionando, dándole, por lo tanto, no sólo al conocimiento que construyamos, sino a la propia práctica del sujeto, un sentido que trascienda las circunstancias más inmediatas.

(E) ¿Te parece que hay una relación fuerte, entre este estrechamiento de la libertad y toda esta ideología, este mito que se hace alrededor de la tecnología? Es decir, el periodismo, la literatura, los trabajos de ficción, el cine, la televisión, dan cuenta de una imagen de la tecnología que nos aplasta. La máquina se viene contra el hombre, la máquina es más poderosa que el hombre, tiene la fuerza y la capacidad de decisión que nosotros no tenemos y que nos va conduciendo hacia el desastre total, hacia el 
caos. Y de tanto escuchar esta versión mitológica, como si la tecnología se pusiera por arriba nuestro y en contra nuestro y fuera más poderosa que nosotros, nos quedamos con una apatía que se ahonda. ¿C ómo reaccionar con libertad frente al mundo tecnológico?

(H Z) La falta de libertad es una de las causas de la apatía, es una de las causas de la desesperanza. Un hombre, un individuo que no encuentra espacio de realización porque no es libre ni siquiera para poder decidir muchas veces sobre lo que es su existencia cotidiana, carece de sentido para él la vida y por lo tanto una forma de expresión que es la indiferencia, la inercia y la apatía. Pero ahora veamos el problema de la tecnología. La tecnología admitiría muchas formas de abordaje, pero quisiera ahora señalar dos dimensiones fundamentales. U na es la de la tecnología como un elemento central de un discurso ideológico hegemónico. La tecnología aparece como siendo el instrumento a través del cual la humanidad mejorará sus condiciones de vida. Ese es el envoltorio con que de alguna manera se hace llegar el mensaje tecnológico a grandes masas humanas. Pero esa dimensión encubre otros aspectos, encubre el hecho de que detrás de la tecnología lo que ocurre es que se disfraza lo que sabemos, una alta concentración de la riqueza. Pero veamos el problema con más detalle.

M uchas veces cuando se dice "el progreso tecnológico"se da por entendido que tiene invariablemente un signo positivo porque el modo de enfrentarmos con el desarrollo tecnológico es excesivamente unilateral o deliberadamente reduccionista. Reducción que impone este discurso que se pretende legitimar precisamnete a través del mensaje tecnológico. Y es reducir el concepto mismo de vida al de condiciones de vida. Es frecuente escuchar, y en los medios de comunicación de masa esto es muy ostensible, cotidianamente, la oferta que se hace a millones de personas de satisfactores, a través de los cuales se les comvence que mejorarán sus condiciones de vida. Se avanza tecnológicamente y el avance tecnológico se presenta como idéntico a la capacidad de satisfacer con mejor calidad, necesidades del hombre. Sin embargo, lo que se oculta, lo que está detrás son otras dimensiones del concepto de la vida normal, cotidiana de la persona como es por ejemplo entender que la vida cotidiana, que la vida del individuo concreto, natural, se resuelve sólo en la dimensión de los satiffactores. Pero muchas veces los satiffactores expresan enajenación, muchas veces los satisfactores, por básicos que éstos sean, son mecanismos también de subalternidad. La gente termina organizando sus vidas y reduciendo el sentido de su vida al alcance de satisfactores. La máxima expresión de esto es el consumismo que se asocia muy directamente con el desarrollo tecnológico. Pero se dejan de lado cosas 
que son tan importantes como el problema de la capacidad del individuo de decidir sobre su propia vida o podríamos Ilamarla, la capacidad de vida que, de alguna manera, expresa, traduce un enriquecimiento de su propia subjetividad. La sociedad, de alguna manera, la sociedad más avanzada, y la no tan avanzada camina, como diría Rudolf Bahro en dirección a transformar al hombre en una hormiga pensante. Eso en el mejor de los casos, pero si subjetividad. La tecnología puede llevar, en la medida en que no tome en cuenta esta dimensión de la subjetividad del individuo y de la capacidad de vida y reducir todo esto simplemente a condiciones de vida a una cultura, aunque esto puede parecer paradojal, a una cultura sin subjetividad, a una cultura sin contenido, solamente con instrumentos. En este contexto de tecnología avanzada, que es un mecanismo de enajenación y también de creación de subalternidad, la libertad a veces, identificándose con la subjetividad del individuo, por lo tanto, mientras más individuo aislado sea, es más libre y a la vez se entiende como una subjetividad más rica. Y ese constituye, sin duda, uno de los engaños porque la subjetividad no la va a encontrar el individuo en su propio estamento, puede vivirlo como individuo, pero no va a encontrar la mayor riqueza de su subjetividad, aislándose de sus relaciones con sus semejantes. Y lo que en este momento se está logrando, a través de ciertos tipos de avance tecnológico, es una creciente soledad de la persona.

(E) M e gustaría que te refirieras al asunto del periodismo, de la ficción? (H Z) La ficción en general, admite juicios diversos, yo diría que la ficción en tanto sea anticipaciones de futuro, creo que puede ser una forma también de estar alerta de la realidad presente. Pero también hay otra ficción, la ficción por ejemplo del consumismo, la ficción de la publicidad, la ficción de la propaganda, esa no muestra un mundo mejor, muestra el mundo actual como el mejor. Entonces, distingamos, un autor de ficción, 0 un periodista que muestra escenarios futuros, o la propia ciencia social, que muestra escanarios futuros sí, por ficticios que sean, en la medida en que permiten ver a distancia lo que vivimos, pueden cumplir una función creativa de alerta. Pero hay otras ficciones, que son las ficciones de transformar la realidad actual en realidades, digamos, en realidades realizadas.

(E) Desde mi punto de vista, la ficción prepara la imagen de que la tecnología es muy poderosa y que el hombre no puede atraparla en su camino, y esto me parece peligroso.

(H Z) Bueno, quiero ver una idea, una idea que puede ser muy ambiciosa en relación a este tópico. Curiosamente la tecnología aparece en este momento como emancipada del hombre, como fuera de la circunstancia 
que es su propia creación. Esto significa precisamente que esa tecnología producto del hombre está siendo en este momento, utilizada por ciertos hombres para mantener a otros hombres en una situación de pasividad, en una situación de indiferencia o de marginalidad. Y aquí, es interesante discutir el concepto que mencionaba yo, el de lo fáustico. Tendríamos que estar viviendo en este momento el período fáustico, de las máximas conquistas, el hombre llega al espacio, el hombre conquista la naturaleza, domina la materia. Sin embargo, ieso es expresión de lo fáustico? Vuelve la impresión de la propia capacidad autodestructiva, porque la idea de Io fáustico, el hombre fáustico era el hombre creador, el hombre prometeico, de alguna manera, pero no estamos en presencia de un hombre prometeico, estamos en presencia de un hombre que ha logrado elaborar una cantidad de inventos, una cantidad de medios para controlar y lograr materializar ese sueño mínimo, pero sin embargo, en el fondo lo condiciona o lo relega a una condición mínima, desplazándose a microespecios, a esos espacios que yo estaba calificando de resistencias, dejando el resto de su espacio social en manos de máquinas o de instrumentos que no necesariamente están emancipados del hombre, sino que están emancipados de ciertos hombres y controlados por ciertos grupos de hombres. Entonces, en ese sentido lo fáustico, esa vieja aspiración se ha reducido a una especie de maquinaria voraz, insaciable, que se está comiendo a sus propios procreadores, a sus propios hijos, pero en beneficio siempre de ciertos grupos, no nos olvidemos de eso. Es decir, la tecnología no escapa al género humano, no escapa a los grupos humanos, utilizada por algunos, para mantener la marginación en aras del progreso de grandes mayorías.

(E) En relación a la pérdida del instinto de conservación, si en todas las épocas ha habido guerras y todo tipo de confrontaciones y de opresión, me pregunto si esta situación de que el hombre se destruye a partir de aquello que él mismo crea, no sería más bien la situación del aprendiz de hechicero que descubre algo, pero que no sabe todo el proceso y no consigue controlar el proceso. Esta cuestión de pérdida del instinto de conservación, ¿no sería tal vez una pérdida de todos los recursos que él tiene y no puede controlar?

(H Z) Puede ser esa una posibilidad de respuesta. Volviendo al viejo mito de Prometeo que robó el fuego a los dioses y que puede destruirlo. Pero yo creo que también habría que ver otras acepciones del problema de la pérdida del instinto. $M$ anejemos una hipóteses, yo creo que el propio desarrollo de la racionalidad en el hombre ha entrado en contradicción con su condición de especie. Es decir, el hombre es la 
única especie que no requiere de un hábitat, que puede generar, crear hábitat, pero esto en un sentido positivo, porque así como puede crear hábitat y trasladarse de un hábitat a otro, también puede destruirlo. Y al destruir esos hábitat también se puede autodestruir. Es decir, aquí hay algo terrible, que naturalmente podría admitir mucha polémica, que es que el crecimiento de la propia inteligencia, en la medida en que crece en el contexto de relaciones sociales desiguales, donde se generan conflictos entre grupos, puede ejercer eventualmente las funciones de un verdadero mutante con respecto a la especie humana y eso no pasa con ninguna otra especie animal, donde rige su comportamiento en base a ciertos instintos, que por definición son preservativos, reproductivos. El hombre no tiene ese instinto, pero el hombre es capaz de modelar, moldear, construir y destruir. Por lo tanto, el lugar donde él pueda reproducirse no está claramente identificado, puede ser cualquiera, pero en ese espacio indiferenciado, como especie puede también destruirse. $\mathrm{N}$ inguna especie destruye su medio ambiente, por definición, ni siquiera en conflicto con otras especies y el hombre es la única especie, precisamente por ese elemento terrible que es su superioridad y a la vez su debilidad, que es la inteligencia, que es en el fondo la capacidad de desprenderse de sus condicionamientos biológicos, de sus condicionamientos naturales, ese mutante que es la inteligencia y la razón hace que el hombre sea capaz de destruir su propio medio ambiente. ¿Por qué? Porque naturalmente, y aquí viene el elemento prometeico, el elemento del aprendiz de brujo, porque viene la esperanza de poder crear siempre un hábitat nuevo donde poder reproducirse. Esto puede llegar incluso hasta el punto de la ficción que estábamos discutiendo, en el sentido de decir, por último, si se destruye la naturaleza, podemos reconstruir la vida en el espacio. Pero evidentemente la reconstrucción de la vida en el espacio no será para la humanidad entera, será sólo para aquellos que controlen la tecnología suficiente para construir ese hábitat en el espacio. Esa lógica está presente de alguna manera. Es decir, el hecho solo de que el hombre se plantee esa hazaña en el fondo es expresión de su propia pérdida de identidad como especie, que está fuertemente asociada a su capacidad de crecer más allá de los condicionamientos naturales que lo definen como tal.

Estamos en presencia de un fenómeno, llamémoslo cultural, que, de seguir así, puede ser ampliamente destructivo. De ahí la importancia de la primera afirmación que yo hice, la capacidad del hombre de poder colocarse ante la exigencia de futuro, desde una visión de futuro definida en términos de valores para poder ver la realidad, reconecer los rumbos 
de la actual realidad y ver en esos rumbos la posibilidad de otras direcciones. Creo que éste es el desafío de la inteligencia bien utilizada en el presente. De otro modo, hay ya una inteligencia mecánica en beneficio de algunos grupos humanos, pero no de toda la humanidad. Podrán salvarse representantes de la especie humana, pero no la humanidad.

(E) Esto me parece muy importante puesto que la solución a la crisis se manifiesta en una la solución elitista y esa la razón por lo que me parece peligroso mirar y aceptar el mensaje del aprendiz de hechicero. La tecnología tiene una posibilidad limitada, pero de tanto oír esto la tecnología domina la vida moderna, está definida por todo. Puedo poner normas y decir qué hago, caer en el cinismo, no hay solución, el mundo no alcanza a luchar contra la tecnología y este es el final de la parada.

(H Z) Exactamente. Y por eso hay que superar la condición de aprendiz de brujo. En ese sentido, yo diría que hay que rescatar algunos elementos que en este momento son importantes. $Y$ esto ya en el plano menos general. Si tratáramos de ver la posibilidad de respuesta en un plano más delimitado, yo diría que gran parte del problema que en este momento se nos presenta supone, entre otras cosas, recuperar una visión utópica, que en el fondo son compromisos con ciertos valores de liberación y que no sean simplemente la proyección del discurso tecnológico. Porque, tomemos conciencia, lo que en este mundo que vivimos, que es un malthusianismo, lo que en este momento se pretende imponer en aras de la razón y en aras del progreso tecnológico es un malthusianismo, y sabemos que eso significa el sacrificio de todos aquellos que no puedan sobrevivir por no ser privilegiados en este momento histórico. En ese plano yo creo que la educación tendría un plano importante que cumplir, que lo sintetizaría en una idea: si la educación no enseña a las nuevas generaciones a pensar el contexto histórico, a ubicarse en el contexto histórico, más allá de las teorías, no podemos esperar que esa gente jovem no haga sino continuar una lógica que en este momento aparece dominada precisamente por el discurso tecnológico. Si queremos que sean capaces de ser protagonistas de un contradiscurso que trate de definir otra dirección de desarrollo para la sociedad, evidentemente para lograrlo esa gente tiene que ser formada de determinada manera, hay que enseñar a pensar el momento y comprometerla con ciertas voluntades de acción que suponen la presencia de valores que permitan avanzar en un sentido de una mayor liberación del conjunto del género humano.

Recebido para publicação em dezembro de 2000. 
TO RECOVER AN UTOPIAN VIEW

(Interview with Prof. Dr. Hugo Zemelman)

ABST RACT: This interview took place in september 23, 1993, when, as Visitor Professor, H ugo Zemelman gave lessonsabout "The reason's horizons and the knowledgés production" at the M aster Course in Education of the U niversidade Federal Fluminense. The interview aim was to know his thought in face of the problems presented by the present and the future of a high tech and sociallly disturbed world.

Key words: Rationality; U topian view; Freedom; Latin America; Future. 